\title{
Display organization and the detection of horizontal line segments
}

\author{
GIAMPAOLO MORAGLIA \\ University of Toronto, Mississauga, Ontario, Canada
}

\begin{abstract}
Observers searched for a horizontal line segment through displays containing varying numbers of elements differing from the target and from each other in terms of orientation. These elements were always positioned on imaginary concentric circles centered in the middle of the display. They were allocated to these positions either randomly or in such a way that their orientation was equal to that of the tangent to the circle at that position. The search for the target line appeared to proceed spatially in parallel with the latter class of displays, and serially with the former. These findings are explained and discussed within the context of the attentive-preattentive dichotomy that characterizes spatial vision.
\end{abstract}

A degree of consensus exists about the hypothesis that certain visual operations can be carried out preattentively (Neisser, 1967)-that is, in parallel across the visual field-and automatically, whereas other operations require the serial allocation of attention to restricted portions of visual space (e.g., Julesz, 1984; Treisman, 1985, 1986). This dichotomy has been extensively investigated within a variety of experimental paradigms (e.g., Treisman \& Gelade, 1980), including visual search. Whether a search is carried out in parallel or through serial processes may also be revealed by the way in which response time varies with the number of nontarget (background) elements in the display. Latencies little affected by increases in the number of background elements are often interpreted as evidence of a parallel search. Increases in response time as the number of elements increases, on the other hand, are regarded as more indicative of a serial search. In particular, if the latencies increase linearly with the number of display elements (display size) and the slope is twice as steep on negative trials (when the target is absent) as on positive trials (when the target is present), the search is then assumed to consist of an attentive, item-by-item analysis of the display, which terminates when the target has been found or when all the items have been checked (e.g., Treisman, 1985, 1986). Whether a search is carried out spatially in parallel or serially is determined chiefly by the nature of the differences between target and background items. No strict unanimity exists, however, about the identity of the stimulus properties that permit the spatially parallel inspection of a display, or about the manner in which this processing mode is implemented by the visual system (cf., e.g., Beck, 1982; Julesz, 1984;

The research presented in this article was supported by grants from the Natural Sciences and Engineering Research Council of Canada, and by a grant from the Connaught Fund. Requests for reprints should be sent to Giampaolo Moraglia, Department of Psychology, Erindale Campus, University of Toronto, Mississauga, Ontario L5L 1C6, Canada.
Marr, 1982; Neisser, 1967; Treisman, 1986; Tsotsos, 1987).

In this study, I attempted to assess if, and to what extent, the mode of a search may also be determined by the spatial distribution of the stimulus property (orientation in this case) upon which the discriminability of the target from the background rests. A number of studies have investigated the degree to which the detectability of simple visual forms may be affected by the spatial context in which these stimuli are presented (e.g., Banks, Bodinger, \& Illige, 1974; Banks \& Prinzmetal, 1976; Prinzmetal \& Banks, 1977; see also Weisstein \& Harris, 1974, and Williams \& Weisstein, 1978). In general, however, these studies were not designed to assess the effects of display configuration on visual search within the context of the attentive-preattentive dichotomy.

In the experiments reported here, observers were asked to search for a short line in a display containing other line segments that differed from the target line and from each other only in terms of orientation. The processing of orientation information has been the subject of intensive investigations by theorists concerned with the conditions that mediate the preattentive inspection of a display (e.g., Beck, 1982; Sagi \& Julesz, 1985, 1987; Treisman, 1985). More generally, in part following the epochal discoveries of Hubel and Wiesel (1962), great efforts have been made to foster our understanding of the manner in which local oriented contours are processed by the visual system (for reviews, see, e.g., De Valois \& De Valois, 1980; Sekuler, 1974; Westheimer, 1984). However, the manner in which the local encoding of orientation information may be related to global, or "field-specific," aspects of an input pattern has received less attention than this issue deserves. Recently, this question was addressed psychophysically by Caelli and Dodwell (1982; Dodwell \& Caelli, 1985; see also Dodwell, 1984), who employed a class of visual displays called vector patterns, or partial representations of a visual vector field. Vector patterns are two-dimensional arrays of short line segments that can 
be described in terms of two (nonindependent) codes. The position code provides the positions on the display where the line segments are. The orientation code specifies the orientation of the line elements at each position. The positions and orientations of the lines can in some cases be related according to simple differential equations, which specify rates of change, or vector orientations, at all points over the two-dimensional field. As an example, the equation $d y / d x=-x / y$ describes the vector field rule for a specific global organization, that of a circular field. With this equation, an orientation $(d y / d x)$ is determined for any specified point in the field, that is, for particular values of $x$ and $y$ (see Dodwell, 1984, p. 231, for an illustration of this formula). The orientations and positions of the line elements give local expression to the overall structure of the field, which is embodied in the differential equation. Two vector patterns, irrespective of the number of line elements, instantiate the same vector field rule as long as every line element in each array obeys the rule. The displays in the upper part of Figure 1, for instance, can all be seen as instances of the circular rule. Caelli and Dodwell (1982) asked observers to discriminate between pairs of vector patterns that were identical except for the introduction of random perturbations of the positions and/or orientations of all the line elements in one member of the pair. They found that the discriminability of these perturbations was related to the vector field rule used to generate the displays, and was highest with patterns embodying a strong organization, such as the circular one. These findings led me to hypothesize that the search for an oriented line segment could be importantly modulated by the organization of the display, and particularly by the orientation distribution function embodied in it. The presence of such an effect, its mode of operation in the context of visual search, and its implications for the attentive-preattentive dichotomy, are the subject of the following experiments.

\section{EXPERIMENT 1}

The choice of the display was inspired by Caelli and Dodwell's (1982) finding that circular vector patterns are among those in which field effects may manifest them-

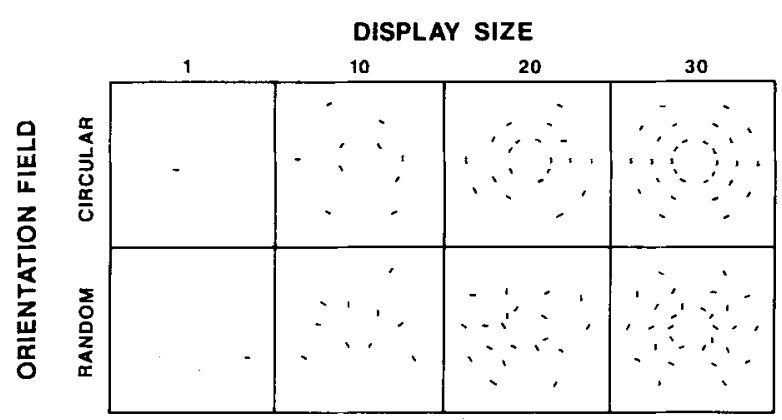

Figure 1. Examples of the vector patterns used in Experiment 1. The target is present in each display. selves with particular strength. As noted, a vector pattern can be specified in terms of orientation and position codes. A circular position field, accordingly, is one in which the elements of the pattern are located on concentric circles centered on the origin of the coordinate system. A vector pattern with circular position code has, in addition, a circular orientation code (thus instantiating the vector field rule described by the differential equation given above) if, at any location, orientation is set to that of the tangent to a circle. Because the focus of these experiments was on the effects of the orientation field on the search for a line target, I employed displays with identical position code (circular) and varied the orientation code, which was either of the circular or of the random type, as described below.

\section{Method}

Subjects. Five observers, 2 males and 3 females, in an age range of 20-38 and with normal or corrected-to-normal visual acuity, served in all experiments. All but one of the observers were naive as to the purpose of the experiments.

Stimuli and Apparatus. The displays, representations of which are given in Fighre 1, were made by hand, with white cards and black ink. In all displays, line segments that each subtended $.67^{\circ}$ of visual angle were drawn in up to 10 positions on each of three imaginary concentric circles centered in the middle of the display area. The diameters of these circles subtended $1.5^{\circ}, 3^{\circ}$, and $4.5^{\circ}$. The 10 positions on each circle were such that the orientations of the tangents to the circle at the midpoints of these positions were $30^{\circ}, 60^{\circ}, 90^{\circ}, 120^{\circ}$, and $150^{\circ}$. The target was a horizontal $\left(0^{\circ}\right)$ line segment (the target is present in each display in Figure 1), and the background elements were in all cases line segments of the above orientations only. Two types of displays were employed. Displays embodying the circular-orientation code ("circular" for brevity) were those in which the background elements were always allocated to positions on the circles in such a way that their orientation was equal to that of the tangent to the circle in that position. With displays whose orientation code was of the random type, the background elements were randomly allocated to these same positions. With both types of displays, four display sizes were used, consisting of $1,10,20$, and 30 elements. For each display type, six cards were made for each combination of the four display sizes and the two levels of target presence (present, absent); thus there were 96 cards. Averaged over a block of trials, consisting of the presentation of all the cards of the random or circular type, the same number of lines appeared on each of the three circles. Also, within a block, the target appeared an equal number of times on each of these circles, and on half of the trials. Within these constraints, the location of the target was selected randomly. The stimuli were displayed via a two-field Cambridge tachistoscope interfaced to a Commodore $\mathbf{8 0 3 2}$ microcomputer that performed display and timing functions. The luminance of the field was $17.1 \mathrm{~cd} / \mathrm{m}^{2}$.

Procedure. On each trial, the observer first fixated a field at whose center was the target line. A tone alerted the observer of the oncoming test display, which was presented $500 \mathrm{msec}$ after the termination of the auditory signal. The onset of the test display started the timing by the computer, which also terminated the display when the observer pressed one of two response keys. The observer's instructions were to decide as quickly and as accurately as possible, by pressing the "yes" or the "no" key, whether the test display had contained the target. A block consisted of 48 correct responses. The number of trials administered during a block thus depended on the observer's error rate, as the test displays to which a subject incorrectly responded were represented later in the 
block. Mean response times (RTs) were computed on the set of correct responses, and on only four of the five blocks that were run for each display type, the first block being treated as practice. This resulted in a total of $48 \times 8=384$ correct responses per observer. The administration of the blocks was independently randomized for each participant.

\section{Results and Discussion}

Mean RT data were analyzed by a four-way, withinsubjects analysis of variance (ANOVA), with subjects, display type (circular and random), trial type (positive and negative), and display size $(1,10,20,30$ elements) as factors. All the main effects were significant [display type, $F(1,4)=26.5, p<.01$; trial type, $F(1,4)=29.3$, $p<.01$; display size, $F(3,12)=40.3, p<.01]$, as were the interactions [display type $\times$ trial type, $F(1,4)=28$, $p<.01$; display type $\times$ display size, $F(3,12)=38.4$, $p<.01$; trial type $\times$ display size, $F(3,12)=18.1$, $p<.01$; display type $\times$ trial type $\times$ display size, $F(3,12)=25.4, p<.01]$. As shown in Figure 2, display size had little effect on RT with the circular vector patterns, whether on positive or on negative trials. Indeed, with this class of displays, the slopes of the regression lines relating display size and RT were not appreciably different from zero for both negative $[t(3)=.88$, $p>.05]$ and positive $[t(3)=1.21, p>.05]$ trials. These findings suggest that the circular displays were searched spatially in parallel. The corresponding slopes were appreciably different from zero with the random displays $[t(3)=31.6, p<.01$, for negative trials; $t(3)=$ $6.9, p<.01$, for positive trials]. Linear regressions of display size on RT for the random vector patterns gave an intercept of $561 \mathrm{msec}$ and a slope of $30 \mathrm{msec}$ for positive trials, and corresponding values of $551 \mathrm{msec}$ and $70 \mathrm{msec}$ for negative trials. The linear component accounted for $96 \%$ of the variance with positive displays, and $100 \%$ with negative displays. The ratio of negative to positive slopes was 2.3. These results are thus more suggestive of a serial, self-terminating search at a rate of about 70 msec per item.

The same ANOVA was performed on the mean standard deviations of RTs. Response time variability increased appreciably with increases in display size $[F(3,12)$ $=32.1, p<.05$ ]; however, as shown in Figure 2, this increase occurred mostly with the displays of the random type [display type, $F(1,4)=18.2, p<.05$; display type and display size, $F(3,12)=27.4, p<.01]$. Generally, variability of RT is greater when RT is greater. In this sense, it is not surprising that the circular displays, by permitting shorter response latencies, also promoted less variability than did the displays of the random type. This finding is also consistent with the hypothesis of a spatially parallel inspection of the circular displays: because the temporal course of this processing mode is not appreciably influenced by display size, the latter should also have a negligible effect on response variability. Mean RT was appreciably greater on negative than on positive trials with the random displays, yet the standard deviation of RT was larger, albeit by a small amount, on positive than on nega- tive trials, and this difference increased with display size. This finding also is compatible with the proposed interpretation of performance. Under the hypothesis of a serial, self-terminating search of these patterns, RT variability should indeed increase on positive trials at least as much as or more than on negative trials, because of the wide range of termination points occurring with the former (see also Donderi, 1983; Krueger, 1984; Krueger \& Shapiro, 1980; Schneider \& Shiffrin, 1977).

On average, errors occurred on $3.9 \%$ of the trials; an ANOVA revealed that errors were appreciably more frequent with random than with circular displays $[F(1,4)=$ $45.6, p<.01]$, and on positive than on negative trials $[F(1,4)=18.9, p<.05]$. Figure 2 shows that they were mostly confined to displays containing more than one element [display size, $F(3,12)=11.3, p<.011$. A significant interaction of display size and trial type $[F(3,12)=$ $10.4, p<.01]$ indicates that the number of elements in the display affected accuracy on positive, but not on negative, trials. Display size also interacted appreciably with display type $[F(3,12)=6.6, p<.01]$; the interaction of display size, display type, and trial type was also significant $[F(3,12)=9.7, p<.01]$. On positive trials, errors increased monotonically with display size with the random, but not with the circular, displays. Performance on negative trials was almost errorless, irrespective of the number of background elements, with both types of display.

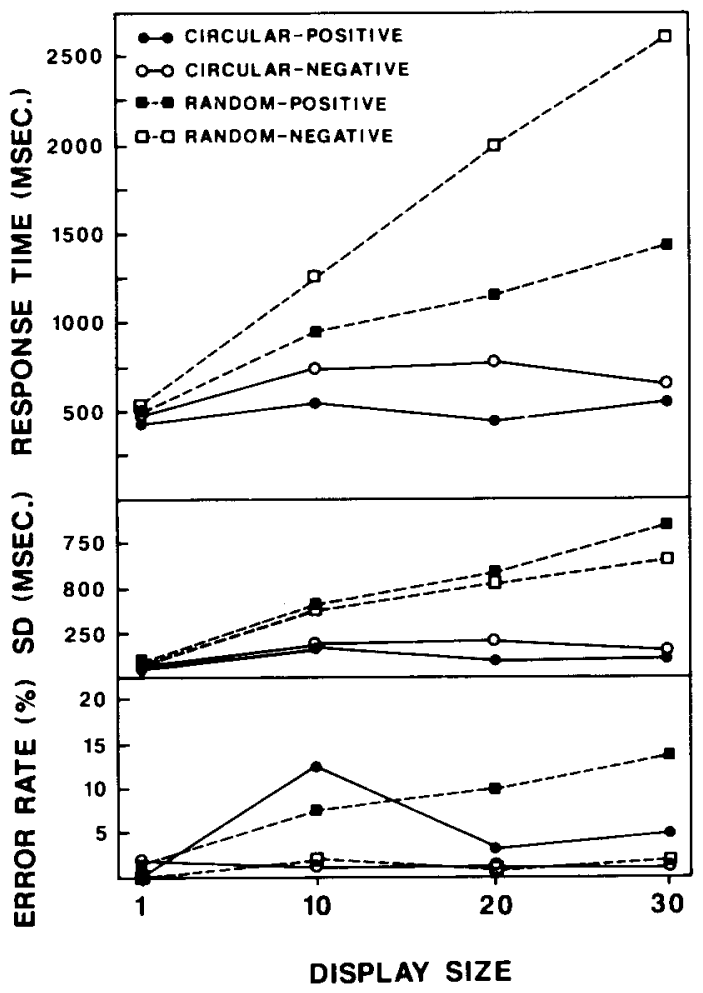

Figure 2. Experiment 1: Mean correct response time, standard deviation $(S D)$ of correct response time, and the percentage of error rate by trial type, display size, and display type. 
On positive trials, with random displays, latencies on incorrect trials were longer than those on the corresponding correct trials $(1,601$ and $1,344 \mathrm{msec}$, respectively). Moreover, RTs on these incorrect trials very closely approximate, at all sizes, the mean values obtained on the negative trials. These findings suggest that false negatives with this class of display likely originated from a failure to correctly identify the target during the item-by-item inspection of the pattern, and that failure resulted in the exhaustive search of the display. Errors with displays of the circular type were, on positive trials, mostly confined to the 10-element displays. In this case as well, latencies on incorrect trials were slightly larger, on average, than those on corresponding correct trials (741 vs. $654 \mathrm{msec}$, respectively), and were close to the mean values for the negative trials $(761 \mathrm{msec})$. I shall argue shortly that the preattentive detection of the target was made possible by the observer's sensitivity to the global organization of these displays. In terms of this hypothesis, it is not surprising that errors were most frequent with the 10-element displays, which instantiated the circular rule more sparsely and thus more weakly than the higher size vector patterns.

The results of this experiment, in summary, show that the structure of the orientation field in which the search was carried out significantly affected performance. When the background elements were allocated to positions according to a circular orientation code, RT was little affected by increases in display size, as with searches carried out preattentively. When the allocation of orientations to positions occurred randomly, latencies increased linearly with increases in the number of background elements and in a manner that appeared to be selfterminating, as with searches that involve serial fixations of attention.

I propose that, with circular displays, the presence of the target was preattentively registered via the detection of a decorrelation between the orientation of the target and the orientation that was expected at the target position on the basis of the curve that best "fits" the pattern's position/orientation code. In other words, the presence of the target was here registered by the detection of a discontinuity in the pattern's circular organization. The absence of a clearly perceived discontinuity, on the other hand, provided the basis for a negative response.

With the random displays, the lack of a structured orientation field precluded the possibility of carrying out the task via the registration of a discontinuity in the regularity of the vector pattern's structure. At each position, the orientation of a line element could take any of six values; in these conditions, a decision about the presence of the target required the positive identification of line orientation. This induced the inspection of the individual pattern elements, which proceeded in a serial, selfterminating manner. This hypothesis, of course, implies that the preattentive identification of line orientation is not possible. Evidence to this effect was indeed obtained by Sagi and Julesz (1985).
The next experiment examined in greater detail the effects of the circular organization on the search for a horizontal line segment.

\section{EXPERIMENT 2}

I suggested that, with circular displays, the detection of a target reduces to the registration of a discontinuity between tangent and actual orientation at some point on the circular position field. One implication of this hypothesis is that the ease with which the presence of the target is reported should then vary directly with the absolute magnitude of the difference between tangent and actual orientation at the target position. Specifically, RT should be longest when the horizontal target is presented at the position whose tangent is also horizontal, and should monotonically decline as the difference between the orientations of the tangent and the target increases, being shortest where the tangent is vertical. This hypothesis was investigated in Experiment 2.

\section{Method}

Only displays of the circular type were employed, and display size was fixed at 30 elements. The target could be present at positions whose tangent orientations were $0^{\circ}, 30^{\circ}, 60^{\circ}, 90^{\circ}, 120^{\circ}$, and $150^{\circ}$. In this experiment, therefore, unlike in Experiment 1, the target was also presented at positions whose tangent orientation was horizontal. Six cards were made for each of the basic positions in which the target could be presented. The allocation of the target to the positions on the three circles was as described in Experiment 1 . In addition to the above, six cards were made that did not contain the target. This resulted in a total of 42 cards, each of which was presented once in a block of trials. Ten such blocks were administered to each observer, for a total of 420 correct responses. The subjects and the procedure were as in the previous experiment.

\section{Results and Discussion}

The results are shown in Figure 3. The data for the $30^{\circ}$ and $150^{\circ}$ (or $-30^{\circ}$ ) orientations are not presented separately since they were very similar; this was also the case for the $60^{\circ}$ and $120^{\circ}$ (or $-60^{\circ}$ ) orientations. The similarity of latencies within these two pairs of orientations agrees well with the hypothesis tested, according to which speed of response should be affected, not by the specific orientation value of the tangent at the target position, but by the absolute magnitude of the difference between the orientations of target and tangent. Also consistent with the hypothesis, latencies were highest when the orientation of the target coincided with that of the tangent to the target position, and decreased monotonically as the difference between the two increased. These data were subjected to a two-way within-subjects ANOVA, with subjects and tangent orientation as factors. The effects of the latter were significant $[F(3,12)=77.6$, $p<.01]$. Tests for linearity of trend revealed the presence of a highly significant linear component $[F(1,12)$ $=198.9, p<.01]$, as well as the presence of a significant quadratic component $[F(1,12)=33.8, p<.01]$. An 


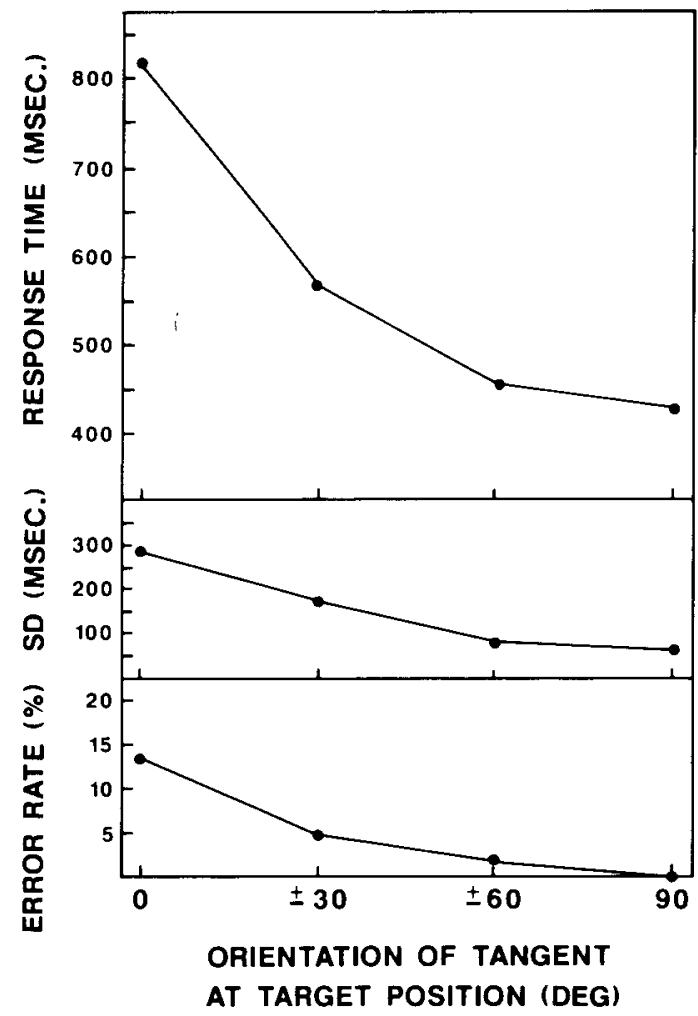

Figure 3. Experiment 2: Mean correct response time, standard deviation $(S D)$ of correct response time, and the percentage of error rate by the orientation of the tangent at the midpoints of the target locations.

$\Omega^{2}$ statistic revealed that the tangent orientation factor accounted for $94 \%$ of the variance; the linear component alone accounted for $81 \%$ of this variance. The mean standard deviations of RTs decreased appreciably $[F(3,12)=$ $40.8, p<.01]$ and monotonically with increases in the difference of tangent and target orientation, and thus with decreases in latency.

An ANOVA also revealed significant variations in response accuracy as a function of tangent orientation $[F(3,12)=17.6, p<.01]$. Errors were most frequent where the orientations of target and tangent coincided, declined as their difference increased, and became zero when the target was presented at the positions whose tangent was vertical. RT on incorrect positive trials was higher than on the corresponding correct trials $(1,037$ and $702 \mathrm{msec}$, respectively), and close to the mean values of the correct negative trials $(1,029 \mathrm{msec})$. False positives were infrequent, averaging $5 \%$ of the negative trials. Latencies on these incorrect trials were close to those obtained on correct positive trials (590 and $572 \mathrm{msec}$, respectively).

The fact that mean latencies on correct positive trials were appreciably lower than on correct negative trials $[t(8)=3.83, p<.01]$ is also to be noted. No such difference was found in Experiment 1 with the 30-element displays of the circular type $[t(8)=.91, p>.05]$. The fact that in Experiment 2 negative trials were less frequent than positive trials could be partially responsible for this difference. It is likely, however, that the increase in RT observed on negative trials also was due to the introduction of the horizontal positions as possible target locations. Correctly reporting the presence of the target at the horizontal positions $(M=822 \mathrm{msec})$ took appreciably longer than at each of the other positions $(p<.01$ with Dunn's tests; the average latency for such positions was $489 \mathrm{msec})$. It also resulted in lower accuracy $(13.3 \%$ and $2.5 \%$ errors for the horizontal and nonhorizontal positions, respectively). The magnitude of these differences raises the possibility that a spatially parallel search of the display did not result in a target "pop out" at the horizontal positions and that its presence there was established instead by an additional attentive inspection of the display at such locations. This hypothesis is compatible with the proposed account of target detection with circular displays. If the latter rested upon the registration of a local decorrelation between tangent and target orientation, this decorrelation did not occur at the horizontal positions. The hypothesis also helps me to explain why latencies on negative trials were higher in Experiment 2 than in the corresponding condition in Experiment $1[t(4)=6.18$, $p<.01]$. In the latter experiment, the observer expected the target to pop out of the display whenever present. The absence of a pop out was thus used by the observer as a criterion for arriving rapidly at a negative response. In Experiment 2, however, the nonoccurrence of a pop out could have been due not only to target absence, but also to the presence of the target at the horizontal locations. The attentive inspection of these locations was thus necessary in both such cases. The hypothesis that the absence of a pop out imposed the continuation of an initially parallel search with a serial search of the horizontal positions, moreover, also explains why, since the latter search is self-terminating, latencies were longer on negative trials than on trials in which the target was present at the horizontal positions [1,029 and $822 \mathrm{msec}$, respectively, $t(4)=4.85, p<.01]$.

\section{GENERAL DISCUSSION}

The above experiments revealed that display organization, as defined by specific position/orientation rules, may powerfully modulate the temporal course of the visual search for an oriented line segment. Consistent with Caelli and Dodwell's findings (1982; Dodwell \& Caelli, 1985), these data confirm the importance of the orientation code in the processing of orientation information. Caelli and Dodwell, who employed a large selection of vector patterns, showed that orientation sensitivity was highest with displays, such as the one used in this study, whose position/orientation codes corresponded to vector fields invariant under the planar transformations of dilation, rotation, or translation. These transformations are among those under which pattern recognition tends to remain invariant (Gibson, 1950, 1966; Hoffman, 1966, 1978). In 
view of their importance, Hoffman (1966; see also Hoffman, 1984; Dodwell, 1970, 1983, 1984) argued for the cortical embodiment of the above vector fields, of which local contour-extraction mechanisms of the type described by Hubel and Wiesel (1962) would be elements. This assumption leads one to expect that vector patterns that instantiate vector field structures of this type should be a particularly easy and natural class of stimuli to process, even for unsophisticated observers. Indeed, vector pattern displays of the circular type employed here, which are rotationally invariant, proved to be salient for the 4month-old infant (see Dodwell, Humphrey, \& Muir, 1987), and similar patterns were found to be particularly easy for the young kitten to discriminate (Dodwell, Wilkinson, \& von Grunau, 1983; Wilkinson \& Dodwell, 1980). The perceptual salience of this type of structure was also made evident by Glass (e.g., 1969; see also Anstis, 1970), who showed that strong circular Moiré fringes could be obtained by superimposing onto itself a random dot pattern rotated by a small amount. Curvaturedependent colored aftereffects were also discussed for their implication with respect to the existence of "curvature detectors"' by Riggs (1973; see also White \& Riggs, 1974) and by MacKay and MacKay (1974). Emerson, Humphrey, and Dodwell (1985) also obtained colored aftereffects contingent on circular and other complex patterns, and argued that these and some other McColloughtype effects are due to visual operations that include the registration of the vector field properties of these patterns.

Regardless of whether or not certain specific vector field rules have a direct cortical representation, all of the studies employing vector patterns have shown that the processing of orientation information in complex displays is improved by these organized structures in comparison with random or weakly organized ones. Within this context, the specific contribution of the present study lies in the suggestion that the presence of a well-formed orientation field may be a necessary condition for the preattentive search of an orientation-defined target in backgrounds of nonhomogeneous orientation.

Experiment 2 showed in particular that the effects of a well-structured orientation field, demonstrated in Experiment 1 , manifest themselves at a local level with the expected degree of strength and regularity. Inspection of Figure 1, however, invites further questions. Consider the circular vector patterns, particularly those with the highest display size. The organizing effect produced by the circular rule can be seen to express itself through various spatial organizations, including rather local ones. An example of the latter is the arrangement of three identical and equidistant line elements perpendicular to a radius passing through their midpoint. More generally, the distribution of vector elements within any subregion of the vector pattern already reveals a strong level of organization. These considerations thus lead to the following question: How are the powerful effects induced by the circular rule distributed at the various spatial scales at which this structure manifests itself? For instance, are these ef- fects mediated most strongly by the local organizations definable in the immediate neighborhood of the target, the contribution of display structure at larger scales progressively declining? This question will be investigated shortly. This question, in turn, raises another concern: Could not these effects be in fact essentially local, and as such independent from the vector field rule embodied in the vector pattern? In Experiment 1, the differences between circular and random vector patterns became evident even at low display sizes; these rules were instantiated by a few elements sparsely distributed over a comparatively wide region of visual space. These findings are thus unsupportive of strictly localistic interpretations. Further experiments under completion in this laboratory, moreover, also suggest a negative answer to the above concern. The local level of organization induced by the linear arrangement of three equally spaced elements of identical orientation, for instance, does not appear to shorten latencies appreciably (in comparison with random vector patterns) when these triplets of elements are allocated to the circles' radii randomly rather than according to the circular rule. If each element of an initially circular vector pattern is allocated to a position on the circle immediately successive (e.g., in a clockwise direction) to the position at which that element's orientation would be identical to the tangent's at that position, the display loses its circular structure; however, any element in both types of display remains surrounded by the same set of orientations. Latencies appear to remain appreciably faster with circular vector patterns than with the other type of display. These findings suggest that, even if they were mediated most strongly by the elements in the vicinity of the target, the effects of the circular organization indeed specifically stem from the manner in which the positions and orientations of the line elements are coordinated by the circular rule.

It is of interest to ask whether these findings are well accommodated by two important views of the attentivepreattentive dichotomy in visual search. Treisman's feature integration theory $(1985,1986$; see also Treisman \& Gelade, 1980; Treisman, 1982) maintains that each of the separable dimensions that define a visual stimulus (e.g., brightness, color, orientation) is internally represented as a stack of "feature maps," each map representing a particular value (feature) along that dimension. The presence of a given feature in the visual field is, accordingly, signaled by activity in the corresponding feature map. A simple model of feature detection can be derived from these assumptions. When looking for a target that uniquely differs from all other items in the display in terms of its value along one basic dimension, such as orientation, the observer monitors the map uniquely activated by the target. Activity, or a change in the level of activity, in that map then leads to a positive response. Lack of activity in the monitored map may, on the other hand, lead to a negative response. According to Treisman, although the observer is granted the opportunity to directly access the output of an individual feature map, 
this output is, however, in the form of a spatially pooled response. The observer can, in other words, determine whether there is activity in a given map, and maybe even the amount of activity, but not where this activity originates or how it is spatially distributed within or between maps. This model, therefore, predicts that a feature target will be preattentively detected whenever it uniquely activates a feature map. Moreover, this should occur independently of the spatial distribution of background items (see also Treisman, 1982), since information about this distribution is not contained in the output that the observer uses as a basis for a response in this search mode.

Of course, for such a model to be directly testable, explicit assumptions should be made about the identity of the various feature maps. Because Treisman's (1986) model, at the present stage of development, does not fully satisfy the latter requirement, a number of theoretical alternatives need to be considered here. If the findings with the circular displays are to be directly interpreted in terms of this model, the existence of a feature map for the horizontal orientation could be assumed. In such a case, an explanation must be given of the reason why this map was not consulted with the displays of the random type. Because the degree of background heterogeneity is the same in both displays, and the spatial distribution of the nontarget elements is unimportant as discussed, I am unable to identify, on the basis of these assumptions, the factors that could have forced a shift to a serial search of the random displays. Recently, Treisman (1985) argued that vertical line targets may not be preattentively coded. Analogously, it could be assumed that no feature maps exists, or is accessible, for the horizontal orientation. Given this assumption, an explanation must then be provided about performance with the circular displays. Treisman's theory is obviously not incompatible with the notion that certain global properties of a display may be detected automatically and in parallel, possibly by largescale global analyzers. As suggested, then, the detection of the target could have occurred via the registration of a local mismatch with the globally perceived structure of the display. This hypothesis, however, entails the simultaneous availability of localized, as well as global, spatial information. Thus, although the model under discussion attempts to account for these findings by postulating the existence of feature maps that signal the presence of certain large-scale properties of the display, it is not clear how information about target-induced local degradations of these same structures could be directly extracted from such maps if their output is assumed to represent spatially pooled activity.

The views of preattentive vision elaborated by Julesz (e.g., 1984) and coworkers are also of importance here. Recently, Sagi and Julesz $(1985,1987)$ showed in particular that detecting and locating orientation gradients can be achieved preattentively, whereas the positive identification of the orientations requires serial fixations of attention. In my experiments, the simple detection and localization of feature gradients could not be a sufficient basis for a response about target presence, given the het- erogeneity of orientations among the background elements. Such a response would likely require the positive identification of the target and thus, if the latter was not preattentively available, the serial search of the display. This theory can thus well account for performance with random displays. It remains to be explained, however, how the recourse to target identification could be avoided in the case of the circular displays, in which the amount of orientation variance was no less than that of the random displays. Sagi and Julesz (1987) also suggested that the preattentive visual system operates only over a short spatial range (about $2^{\circ}$ ). The insensitivity of the preattentive visual system to long-range spatial interactions, then, discourages an explanation of performance with circular vector patterns similar to that suggested here. Rather, an account of these findings ought to be provided in terms of strictly local spatial interactions among line detectors, of the type recently outlined by these authors (Sagi \& Julesz, 1987). It is difficult to see, however, how such interactions could easily account for all the effects discussed here.

The accommodation of the effects of display organization in the current characterization of the preattentive-attentive dichotomy, and within the domain of the visual search for orientation-defined targets, may thus require closer theoretical and experimental scrutiny. This certainly applies to the approach outlined in the present study, in which only two basic orientation codes were probed, and in rather preliminary terms. Important questions need to be answered about which orientation codes promote, and which inhibit, the preattentive search of an orientationdefined target. Interesting results will also likely arise from an equally systematic exploration of position codes, and from an improved understanding of the relation between the two codes as captured by different vector field rules. Some of these issues will be addressed in a subsequent paper.

\section{REFERENCES}

ANSTIS, S. (1970). Phi movement as a subtraction process. Vision Research, 10, 1411-1430.

Banks, W., Bodinger, D., \&lluge, M. (1974). Visual detection acuity and target-noise proximity. Bulletin of the Psychonomic Society, 4 , $411-414$

Banks, W., \& Prinzmetal, W. (1976). Configurational effects in visual information processing. Perception \& Psychophysics, 19, 361-367.

BECK, J. (1982). Textural segmentation. In J. Beck (Ed.), Organization and representation in perception (pp. 285-317). Hillsdale, $\mathrm{NJ}$ : Erlbaum.

Caeld, T., \& Dodwell, P. C. (1982). The discrimination of structure in vectorgraphs: Local and global effects. Perception \& Psychophysics, 32, 314-326.

De Valois, R. R., De Valoss, K. K. (1980). Spatial vision. Annual Review of Psychology, 31, 309-341.

Dodwell, P. C. (1970). Visual pattem recognition. New York: Holt, Rinehart \& Winston.

DODWELL, P. C. (1983). The Lie transformation group model of visual perception. Perception \& Psychophysics, 34, 1-16.

DoDWELL, P. C. (1984). Local and global factors in figural synthesis. In P. C. Dodwell \& T. Caelli (Eds.), Figural synthesis (pp. 219-248). Hillsdale, NJ: Erlbaum.

Dodwell, P. C., CAELLI, T. (1985). Recognition of vector pattems 
under transformation: Local and global determinants. Quarterly Journal of Experimental Psychology, 37, 1-23.

Dodwell, P. C., Humphrey, G. K., \& Muir, D. W. (1987). Shape and pattern perception. In P. Salapatek \& L. Cohen (Eds.), Handbook of infant perception (Vol. 2, pp. 1-77). New York: Academic Press.

Dodwell, P. C., Wilkinson, D., \& von Grunau, M. (1983). Pattern recognition in kittens: Performance on Lie patterns. Perception, 12. 393-410.

DONDERI, D. C. (1983). Acquisition and decision in visual same-different search of letter displays. Perception \& Psychophysics, 33, 271-282.

Emerson, D., Humphrey, G. K., \& Dodwell, P. C. (1985). Colored aftereffects contingent on patterns generated by Lie Transformations Groups. Perception \& Psychophysics, 37, 155-162.

GiBson, J. J. (1950). The perception of the visual world. Boston: Houghton Mifflin.

GiBson, J. J. (1966). The senses considered as perceptual systems. Boston: Houghton Mifflin.

Glass, L. (1969). Moiré effects from random dots. Nature, 223, 578-580.

Hoffman, W. C. (1966), The Lie algebra of visual perception. Journal of Mathematical Psychology, 3, 65-98.

HoffmaN, W. C. (1978). The Lie Transformation Group approach to visual neuropsychology. In H. Leeuwenberg \& H. Buffart (Eds.), Formal theories of visual perception (pp. 30-79). New York: Wiley.

Hoffman, W. C. (1984). Figural synthesis by vector fields: Geometric neurophsychology. In P. C. Dodwell \& T. Caelli (Eds.), Figural synthesis (pp. 249-282). Hillsdale, NJ: Erlbaum.

Hubel, D. H., \& WiESEL, T. N. (1962). Receptive fields, binocular interactions, and functional architecture in the cat's visual cortex. Journal of Physiology, 160, 106-154.

JULESZ, B. (1984). Toward an axiomatic theory of preattentive vision. In G. M. Edelman, W. E. Gall, \& W. M. Cowan (Eds.), Dynamic aspects of neocortical functions (pp. 585-612). New York: Wiley.

KRUEGER, L. E. (1984). Self-termination in same-different judgements: Multiletter comparison with simultaneous and sequential presentation. Journal of Experimental Psychology: Learning, Memory, \& Cognition, 10, 271-284.

Krueger, L. E., \& Shapiro, L. G. (1980). Why search for target absence is so slow (and careful!): The more targets there are, the more likely you are to miss one. Journal of Experimental Psychology: Human Perception \& Performance, 6, 662-685.

MACKAY, D. M., \& MACKAY, V. (1974). Do curvature-contingent chromatic aftereffects require "detectors for curvature"? Vision Research, 14, 1285-1287
Marr, D. (1982), Vision. San Francisco: W. H. Freeman.

Neisser, U. (1967). Cognitive psychology. New York: AppletonCentury-Crofts.

Prinzmetal, W., \& Banks, W. P. (1977). Good continuation affects visual detection. Perception \& Psychophysics, 21, 389-395

RigGs, L. A. (1973). Curvature as a feature of pattern vision. Science, 181, 1070-1072.

SAGI, D., \& Julesz, B. (1985). 'Where' and 'What' in vision. Science, 228, 1217-1219.

SAGI, D., \& JULESZ, B. (1987). Short-range limitation on detection of feature differences. Spatial Vision, 2, 39-51.

SChNeIDER, W., \& Shiffrin, R. M. (1977). Controlled and automatic human information processing: I. Detection, search, and attention. Psychological Review, 84, 1-66.

SEKULER, R. (1974). Spatial vision. Annual Review of Psychology, 25 , 195-232.

Treisman, A. (1982). Perceptual grouping and attention in visual search for features and objects. Journal of Experimental Psychology: $\mathrm{Hu}$ man Perception \& Performance, 8, 194-214.

Treisman, A. (1985). Preattentive processing in vision. Computer Vision, Graphics, \& Image Processing, 31, 156-177.

Treisman, A. (1986). Features and objects in visual processing. Scientific American, 255, 114B-125.

Treisman, A., \& Gelade, G. (1980). A feature-integration theory of attention. Cognitive Psychology, 12, 97-136.

Tsotsos, J. (1987). Analyzing vision at the complexity level: Constraints on an architecture, an explanation for visual search performance, and computational justification for attentive processes. Technical Reports on Research in Biological and Computational Vision at the University of Toronto, No. 20.

Weisstein, N., \& HarrIs, C. S. (1974). Visual detection of line segments: An object superiority effect. Science, 186, 752-755.

Westheimer, G. (1984). Spatial vision. Annual Review of Psychology, 35, 201-226.

White, K. D., \& RigGS, L. A. (1974). Angle-contingent color aftereffects. Vision Research, 14, 1147-1154.

Wilkinson, F. W., \& Dodwell, P. C. (1980). Young kittens can learn complex visual pattern discrimination. Nature, 284, 258-259.

Williams, A., \& Weisstein, N. (1978). Line segments are perceived better in a coherent context than alone: An object-line effect in visual perception. Memory \& Cognition, 6, 85-90.

(Manuscript received April 4, 1988; revision accepted for publication August 12, 1988.) 